\title{
LOCALLY COMPACT SEMILOCAL RINGS
}

\author{
BY SETH WARNER ${ }^{1}$
}

Communicated by Bertram Yood, May 19, 1967

We seek to determine when a locally compact ring is the topological direct product of finitely many algebras over locally compact fields; here we shall consider only commutative rings with identity.

A semilocal ring is a commutative ring with identity that possesses only finitely many maximal ideals. A local ring is a commutative ring with identity that possesses only one maximal ideal (in particular, we do not require that a local ring be noetherian nor that the intersection of the powers of its maximal ideal be the zero ideal). An equicharacteristic ring is a commutative ring with identity $A$ such that for every maximal ideal $\mathfrak{m}$ of $A, A / \mathfrak{m}$ has the same characteristic as $A$. An algebra over a field is a Cohen algebra if it is a local algebra whose maximal ideal has codimension one.

Theorem 1. If $A$ is a commutative Hausdorff topological ring with identity, then $A$ is a locally compact equicharacteristic semilocal ring none of whose maximal ideals is open if and only if $A$ is the topological direct product of finitely many finite-dimensional Cohen algebras over indiscrete locally compact fields that have the same characteristic.

Outline of Proof. The condition is clearly sufficient. In proving that the condition is necessary, we establish first that the invertible elements of $A$ form an open set, that inversion is continuous where defined, and consequently that every maximal ideal of $A$ is closed. For this, theorems of Kaplansky [3, Theorem 6, Lemma 3] enable us to assume that $A$ is totally disconnected; Kaplansky's characterization of compact semisimple rings [2, Theorem 16] yields the result in this case.

Next, we consider the case where $A$ is totally disconnected (if $A$ has prime characteristic, the theory of characters implies that $A$ is necessarily totally disconnected). If $A$ has characteristic zero, then $A$ contains a subfield $Q$ algebraically isomorphic to the field of rationals; the open additive subgroups of $Q$ form a fundamental system of neighborhoods of zero for the induced topology on $Q$, which is indiscrete. Similarly, if $A$ has prime characteristic, then $A$ contains a field $P(a)$ algebraically isomorphic to the field of rational functions over a finite field $P$; the open $P[a]$-submodules of $P(a)$ form a fundamental system of neighborhoods of zero for the induced topology on $P(a)$, which is indiscrete. Consequently in both cases we may apply

\footnotetext{
1 Research supported by NSF grant GP 5933.
} 
the following theorem of $\mathrm{E}$. Correl [1]: If $K$ is the quotient field of a principal ideal domain $A$ and if $J$ is an indiscrete Hausdorff topology on $K$ for which $K$ is a topological field, then the open $A$-submodules of $K$ form a fundamental system of neighborhoods of zero for $I$ if and only if $\Im$ is the supremum of a family of $p$-adic topologies on $K$ (i.e., topologies defined by $p$-adic valuations, where $p$ is a prime of $A$ ). It follows easily that $A$ is the topological direct product of finitely many (necessarily finite-dimensional) algebras over indiscrete locally compact fields; each such algebra has a nilpotent radical, and consequently by raising idempotents we may assume that each algebra is actually a local algebra. Using what we have proved thus far and making suitable changes in the proof of I. S. Cohen's theorem on equicharacteristic local rings as presented in [6, pp. 304-305], we may show that each locally compact local ring that contains an indiscrete locally compact field $K$ is a Cohen algebra over a locally compact extension of $K$. This completes the proof of the theorem for the case where $A$ is totally disconnected.

If $A$ is connected, the theorem is established by use of the Pontrjagin-van Kampen theorem [4, p. 110] on the structure of abelian locally compact groups and a theorem of Kaplansky [3, Theorem 1] on connected additive subgroups of locally compact rings.

In the general case, we may use what has been proved thus far to show that the radical of $A$ is nilpotent. Hence by raising idempotents, we may assume that $A$ is a local ring whose maximal ideal is nilpotent. But in this case, standard theorems concerning locally compact vector spaces over indiscrete locally compact fields establish that $A$ is either connected or totally disconnected, and the proof is complete.

Following Bourbaki, we shall call a Hausdorff topological ring a Polish ring if it is complete and possesses a countable base for the open sets.

THEOREM 2. Let $A$ be a commutative Hausdorff topological ring with identity, and let $p$ be either zero or a prime. Then $A$ is a Polish, locally compact, equicharacteristic artinian ring of characteristic $p$ if and only if $A$ is the topological direct product of finitely many finite-dimensional Cohen algebras over indiscrete locally compact fields of characteristic $p$ and finitely many discrete finite-dimensional Cohen algebras over discrete countable fields of characteristic $p$.

OutLine of Proof. The condition is clearly sufficient since indiscrete locally compact fields and countable discrete fields are Polish fields. Necessity: By algebraic theorems concerning commutative artinian rings [5, Theorem 3, p. 205], we may assume that $A$ is local; its maximal ideal $\mathfrak{m}$ is then necessarily nilpotent. By Theorem 1 we 
need only consider the case where $\mathfrak{m}$ is open. A category argument establishes that if $\mathfrak{b}$ is a finitely generated open ideal of a Polish locally compact commutative ring, then all powers of $\mathfrak{b}$ are open. Consequently, $A$ is discrete, and the proof is complete.

It is easy to construct an example to show that a locally compact equicharacteristic artinian ring that is not Polish need not be the topological direct product of algebras over indiscrete locally compact fields and discrete algebras over discrete fields.

THEOREM 3. Let $A$ be a commutative Hausdorff topological ring with identity. Then $A$ is a Polish, locally compact, equicharacteristic, semilocal noetherian ring of characteristic zero if and only if $A$ is the topological direct product of finitely many finite-dimensional Cohen algebras over indiscrete locally compact fields of characteristic zero and (possibly) a countable discrete semilocal noetherian algebra over the discrete topological field $\boldsymbol{Q}$ of rationals.

Outline of Proof. If $\mathfrak{a}$ is an ideal of a commutative noetherian ring, we denote the intersection of the powers of $a$ by $\mathfrak{a}^{\prime}$, and we define $\mathfrak{a}^{(n)}$ inductively by $\mathfrak{a}^{(0)}=\mathfrak{a}, \mathfrak{a}^{(k+1)}=\left(\mathfrak{a}^{(k)}\right)^{\prime}$. A theorem on commutative noetherian rings [5, Corollary, p. 218] may be used to show that $\left(\mathfrak{a}^{(n)}\right)_{n \geq 0}$ is a decreasing stationary sequence. If $\mathfrak{a}$ is an open ideal of $A$, the hypothesis that $A$ is equicharacteristic of characteristic zero implies that $\mathfrak{a}^{\prime}$ is open. Combining these two results, we obtain a decomposition of $A$ into the direct product of an open ideal none of whose maximal ideals is open and a discrete ideal. An application of Theorem 1 completes the proof.

One may easily show that the hypothesis that $A$ be Polish cannot be omitted. Moreover, the hypothesis that $A$ have characteristic zero cannot be replaced by the hypothesis that $A$ have prime characteristic, for the valuation ring of an indiscrete locally compact field of prime characteristic is a Polish, compact, local principal ideal domain, but it is not an algebra over any indiscrete locally compact field.

\section{REFERENCES}

1. Ellen Correl, On topologies for fields, $\mathrm{Ph}$.D. thesis, Purdue University, Lafayette, Indiana, 1958.

2. Irving Kaplansky, Topological rings, Amer. J. Math. 69 (1947), 153-183.

3. - Locally compact rings, Amer. J. Math. 70 (1948), 447-459.

4. André Weil, L'intégration dans les groupes topologiques et ses applications, Hermann, Paris, 1938.

5. Oscar Zariski and Pierre Samuel, Commutative algebra, vol. I, van Nostrand, Princeton, N. J., 1958.

6. - Commutative algebra, vol. II, van Nostrand, Princeton, N. J., 1960.

DUKe UnIVERSITY 